Network Working Group

Request for Comments: 3712

Category: Informational

P. Fleming

IBM

I. McDonald

High North

February 2004

\title{
Lightweight Directory Access Protocol (LDAP): Schema for Printer Services
}

Status of this Memo

This memo provides information for the Internet community. It does not specify an Internet standard of any kind. Distribution of this memo is unlimited.

Copyright Notice

Copyright (C) The Internet Society (2004). All Rights Reserved.

Abstract

This document defines a schema, object classes and attributes, for printers and printer services, for use with directories that support Lightweight Directory Access Protocol v3 (LDAP-TS). This document is based on the printer attributes listed in Appendix E of Internet Printing Protocol/1.1 (IPP) (RFC 2911). A few additional printer attributes are based on definitions in the Printer MIB (RFC 1759).

Table of Contents

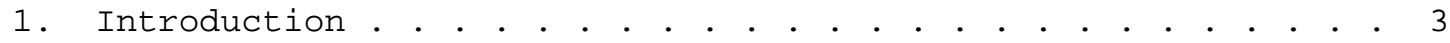

1.1. Rationale for using Directorystring Syntax . . . . . . 3

1.2. Rationale for using caselgnoreMatch. . . . . . . . . . 4 4

1.3. Rationale for using caseIgnoreSubstringsMatch. • • • . 5

2. Terminology and Conventions. • • • • • • • • • • • • . • • . . 5

3. Definition of Object Classes . . . . . . . . . . . . . . 6

3.1. slpserviceprinter. . . . . . . . . . . . . . . 6

3.2. printerAbstract. . . . . . . . . . . . . . . . 7

3.3. printerService . . . . . . . . . . . . . . . . . . 8

3.4. printerServiceAuxClass . . . . . . . . • . • . . . 8

3.5. printerIPP . . . . . . . . . . . . . . . . . . . . . . 8

3.6. printerLPR . . . . . . . . . . . . . . . . . . . . . 9

4. Definition of Attribute Types. • . . . . . . • • • • . . . . . 9

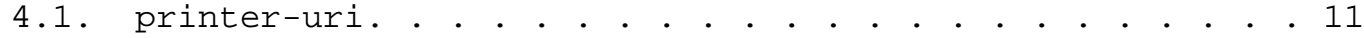

4.2. printer-xri-supported. . . . . . . . . . . . . . . . 11

4.3. printer-name . . . . . . . . . . . . . . . . . . . . . . . .913

4.4. printer-natural-language-configured. . . . . . . . . 13 
4.5. printer-location . . . . . . . . . . . . . . . . . 14

4.6. printer-info. . . . . . . . . . . . . . . . . . . . . 14

4.7. printer-more-info. . . . . . . . . . . . . . . . . . . . 14

4.8. printer-make-and-model . . . . . . . . . . . . . 15

4.9. printer-ipp-versions-supported . . . . . . . . . . . . 15

4.10. printer-multiple-document-jobs-supported . . . . . . . 16

4.11. printer-charset-configured . . . . . . . . . . . . 16

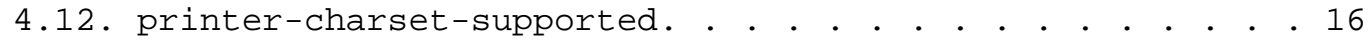

4.13. printer-generated-natural-language-supported • • • • • 17

4.14. printer-document-format-supported. . . . . . . . . . . 17

4.15. printer-color-supported. . . . . . . . . . . . . . . . 18

4.16. printer-compression-supported. . . . . . . . . . . . . 18

4.17. printer-pages-per-minute . . . . . . . . . . . . . . 18

4.18. printer-pages-per-minute-color . . . . . . . . . . . . 19

4.19. printer-finishings-supported . . . . . . . . . . . . . . 19

4.20. printer-number-up-supported. . . . . . . . . . . . . . 19

4.21. printer-sides-supported. . . • . • • • • • • • • • . 20

4.22. printer-media-supported. . . . . . . . . . . . . . 20

4.23. printer-media-local-supported. . . . . . . . . . . . . . 20

4.24. printer-resolution-supported . . . . . . . . . . . . . . 21

4.25. printer-print-quality-supported. . . . . • • • • • • . 22

4.26. printer-job-priority-supported . . . . . . . . . . . . . 22

4.27. printer-copies-supported . . . . . . . . . . . . . . . 22

4.28. printer-job-k-octets-supported . . . . . . . . • . . . 23

4.29. printer-current-operator . . . . . . . . . . . . . 23

4.30. printer-service-person . . . . . . . . . . . . . . 24

4.31. printer-delivery-orientation-supported . . . . . . . . . 24

4.32. printer-stacking-order-supported . . . . . . . . . . . 24

4.33. printer-output-features-supported. • • • • • • • • • • 25

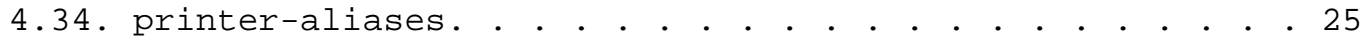

5. Definition of Syntaxes . . . . . . . . . . . . . . . 26

6. Definition of Matching Rules . . . . . . . . . . . . . 26

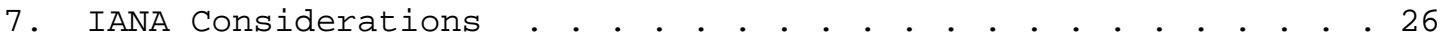

7.1. Registration of Object Classes . • . • . • . . . . . . 26

7.2. Registration of Attribute Types. . . . . . . . . . . . 27

8. Internationalization Considerations. . . . . . . . . . . . 28

9. Security Considerations. . . . . . . . . . . . . . . . 29

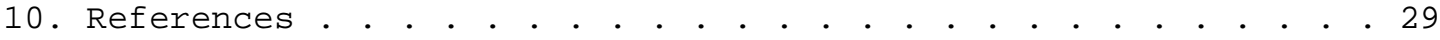

10.1. Normative References . . . . . . . . . . . . . . . 29

10.2. Informative References . . . . . . . . . . . . . . . 30

11. Acknowledgments. . . . . . . . . . . . . . . . . . . 32

12. Authors' Addresses . . . . . . . . . . . . . . . . . . . 32

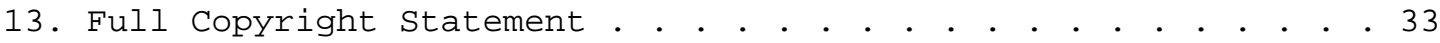




\section{Introduction}

This document defines several object classes to provide Lightweight Directory Access Protocol v3 [LDAP-TS] applications with flexible options in defining printer information using LDAP schema. Classes are provided for defining directory entries with common printer information as well as for extending existing directory entries with SLPv2 [RFC2608], IPP/1.1 [RFC2911], and LPR [RFC1179] specific information.

The schema defined in this document is based on the printer attributes listed in Appendix E 'Generic Directory Schema' of Internet Printing Protocol/1.1 (IPP) [RFC2911]. A few additional printer attributes are based on definitions in the Printer MIB [RFC1759].

The schema defined in this document is technically aligned with the stable IANA-registered 'service:printer:' v2.0 template [SLP-PRT], for compatibility with already deployed Service Location Protocol (SLPV2) [RFC2608] service advertising and discovery infrastructure. The attribute syntaxes are technically aligned with the 'service:printer:' v2.0 template - therefore simpler types are sometimes used (for example, 'Directorystring' [RFC2252] rather than 'labeledURI' [RFC2079] for the 'printer-uri' attribute).

Please send comments directly to the authors at the addresses listed in Section 13 "Authors' Addresses".

1.1. Rationale for using Directorystring Syntax

The attribute syntax 'DirectoryString' (UTF-8 [RFC2279]) defined in [RFC2252] is specified for several groups of string attributes that are defined in this document:

1) URI

- printer-uri, printer-xri-supported, printer-more-info

The UTF-8 encoding is forward compatible with any future deployment of (UTF-8 based) IRI (Internationalized Resource Identifiers) [W3C-IRI] currently being developed by the W3C Internationalization Working Group.

2) Description

- printer-name, printer-location, printer-info, printer-make-and-model 
The UTF-8 encoding supports descriptions in any language, conformant with the "IETF Policy on Character sets and Languages" [RFC2277].

Note: The printer-natural-language-configured attribute contains a language tag [RFC3066] for these description attributes (for example, to support text-to-speech conversions).

3) Keyword

- printer-compression-supported, printer-finishings-supported, printer-media-supported, printer-media-local-supported, printer-print-quality-supported

The UTF-8 encoding is compatible with the current IPP/1.1 [RFC2911] definition of the equivalent attributes, most of which have the IPP/1.1 union syntax 'keyword or name'. The keyword attributes defined in this document are extensible by site-specific or vendor-specific 'names' which behave like new 'keywords'

Note: In IPP/1.1, each value is strongly typed over-the-wire as either 'keyword' or 'name'. This union selector is not preserved in the definitions of these equivalent LDAP attributes.

\subsection{Rationale for using caseIgnoreMatch}

The EQUALITY matching rule 'caseIgnoreMatch' defined in [RFC2252] is specified for several groups of string attributes that are defined in this document:

1) URI

These URI attributes specify EQUALITY matching with

'caseIgnoreMatch' (rather than with 'caseExactMatch') in order to conform to the spirit of [RFC2396], which requires case insensitive matching on the host part of a URI versus case sensitive matching on the remainder of a URI.

These URI attributes follow existing practice of supporting case insensitive equality matching for host names in the associatedDomain attribute defined in [RFC1274].

Either equality matching rule choice would be a compromise: a) case sensitive whole URI matching may lead to false negative matches and has been shown to be fragile (given deployed client applications that 'pretty up' host names displayed and transferred in URI); 
b) case insensitive whole URI matching may lead to false positive matches, although it is a dangerous practice to publish URI that differ only by case (for example, in the path elements).

2) Description

Case insensitive equality matching is more user-friendly for description attributes.

3) Keyword

Case insensitive equality matching is more user-friendly for keyword attributes.

1.3. Rationale for using caselgnoreSubstringsMatch

The SUBSTR matching rule 'caseIgnoreSubstringsMatch' defined in [RFC2252] is specified for several groups of string attributes that are defined in this document:

1) URI

These URI attributes follow existing practice of supporting case insensitive equality matching for host names in the associatedDomain attribute defined in [RFC1274].

2) Description

Support for case insensitive substring matching is more user-friendly for description attributes.

3) Keyword

Support for case insensitive substring matching is more user-friendly for keyword attributes.

2. Terminology and Conventions

The key words "MUST", "MUST NOT", "REQUIRED", "SHALL", "SHALL NOT", "SHOULD", "SHOULD NOT", "RECOMMENDED", "MAY", and "OPTIONAL" in this document are to be interpreted as described in BCP 14 [RFC2119].

Schema definitions are provided using LDAPv3 [LDAP-TS] description formats. Definitions provided here are formatted (line wrapped) for readability. 
3. Definition of Object Classes

We define the following LDAP object classes for use with both generic printer related information and services specific to SLPV2 [RFC2608], IPP/1.1 [RFC2911], and LPR [RFC1179].

slpserviceprinter - auxiliary class for SLP registered printers printerAbstract - abstract class for all printer classes printerService - structural class for printers printerServiceAuxClass - auxiliary class for printers printerIPP - auxiliary class for IPP printers printerLPR - auxiliary class for LPR printers

The following are some examples of how applications may choose to use these classes when creating directory entries:

1) Use printerService for directory entries containing common printer information.

2) Use both printerService and slpServiceprinter for directory entries containing common printer information for SLP registered printers.

3) Use printerService, printerLPR and printerIPP for directory entries containing common printer information for printers that support both LPR and IPP.

4) Use printerServiceAuxclass and object classes not defined by this document for directory entries containing common printer information. In this example, printerServiceAuxclass is used for extending other structural classes defining printer information with common printer information defined in this document.

Refer to Section 4 for definition of attribute types referenced by these object classes. We use attribute names instead of OIDs in object class definitions for clarity. Some attribute names described in [RFC2911] have been prefixed with 'printer-' as recommended in [RFC2926] and [SLP-PRT].

3.1. slpserviceprinter

$(1.3 .18 \cdot 0.2 \cdot 6.254$

NAME 'slpServicePrinter'

DESC 'Service Location Protocol (SLP) information.' AUXILIARY

SUP slpservice ) 
This auxiliary class defines Service Location Protocol (SLPv2) [RFC2608] specific information. It should be used with a structural class such as printerservice. It may be used to create new or extend existing directory entries with SLP 'service:printer' abstract service type information as defined in [SLP-PRT]. This object class is derived from 'slpservice', the parent class for all SLP services, defined in [RFC2926].

\section{2. printerAbstract}

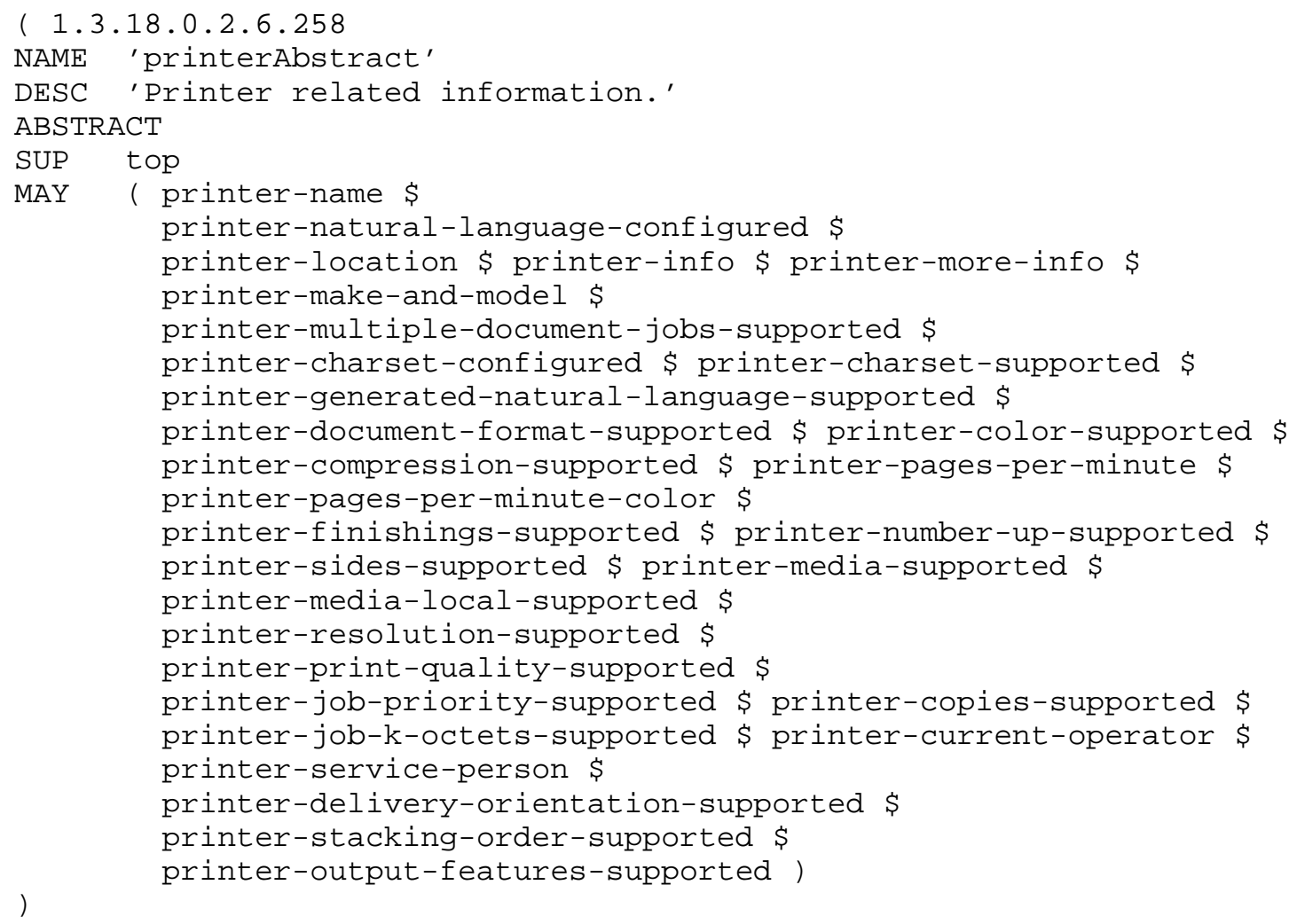

This abstract class defines printer information. It is a base class for deriving other printer related classes, such as, but not limited to, classes defined in this document. It defines a common set of printer attributes that are not specific to any one type of service, protocol or operating system. 


\section{3. printerService}

$(1.3 .18 .0 .2 .6 .255$

NAME 'printerService'

DESC 'Printer information.'

STRUCTURAL

SUP printerAbstract

MAY ( printer-uri \$ printer-xri-supported )

)

This structural class defines printer information. It is derived from class printerAbstract and thus inherits common printer attributes. This class can be used with or without auxiliary classes to define printer information. Auxiliary classes can be used to extend the common printer information with protocol, service or operating system specific information.

Note: When extending other structural classes with auxiliary classes, printerService should not be used.

3.4. printerServiceAuxClass

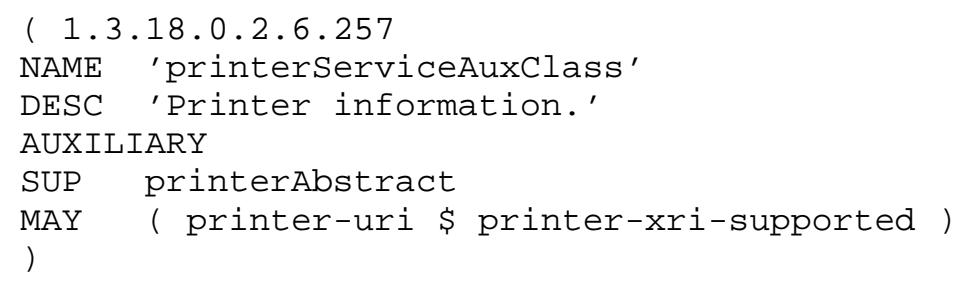

This auxiliary class defines printer information. It is derived from class printerAbstract and thus inherits common printer attributes. This class should be used with a structural class.

\section{5. printerIPP}

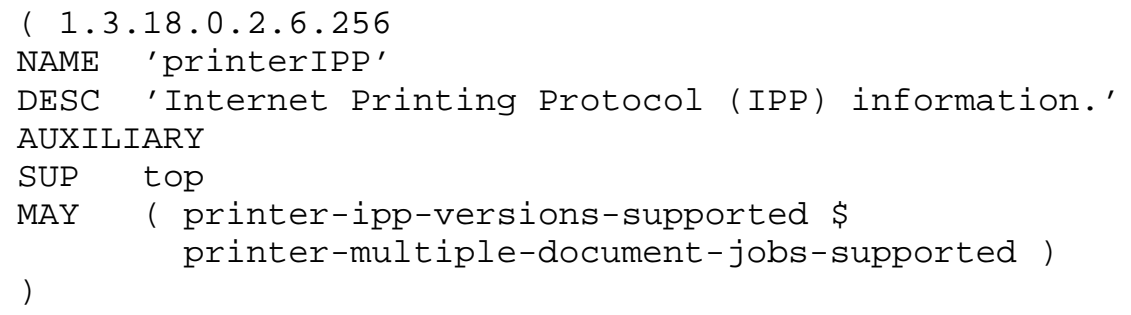


This auxiliary class defines Internet Printing Protocol (IPP/1.1) [RFC2911] information. It should be used with a structural class such as printerservice. It is used to extend structural classes with IPP specific printer information.

3.6. printerLPR

$(1.3 .18 \cdot 0.2 \cdot 6.253$

NAME 'printerLPR'

DESC 'LPR information.'

AUXILIARY

SUP top

MUST ( printer-name )

MAY ( printer-aliases)

)

This auxiliary class defines LPR [RFC1179] information. It should be used with a structural class such as printerService. It is used to identify directory entries that support LPR.

4. Definition of Attribute Types

The following attribute types are referenced by the object classes defined in section 3.

The following attribute types reference syntax OIDs defined in Section 6 of [RFC2252] (see Section 5 'Definition of Syntaxes' below).

The following attribute types reference matching rule names (instead of OIDs) for clarity (see section 6 below). For optional attributes, if the printer information is not known, the attribute value should not be set. In the following definitions, referenced matching rules are defined in Section 8 of [RFC2252] and/or Section 2 of [RFC3698] (see Section 6 'Definition of Matching Rules' below).

The following table is a summary of the attribute names defined by this document and their corresponding names from [RFC2911]. Some attribute names described in [RFC2911] have been prefixed with 'printer-' as recommended in [RFC2926], to address the flat namespace for LDAP identifiers. 


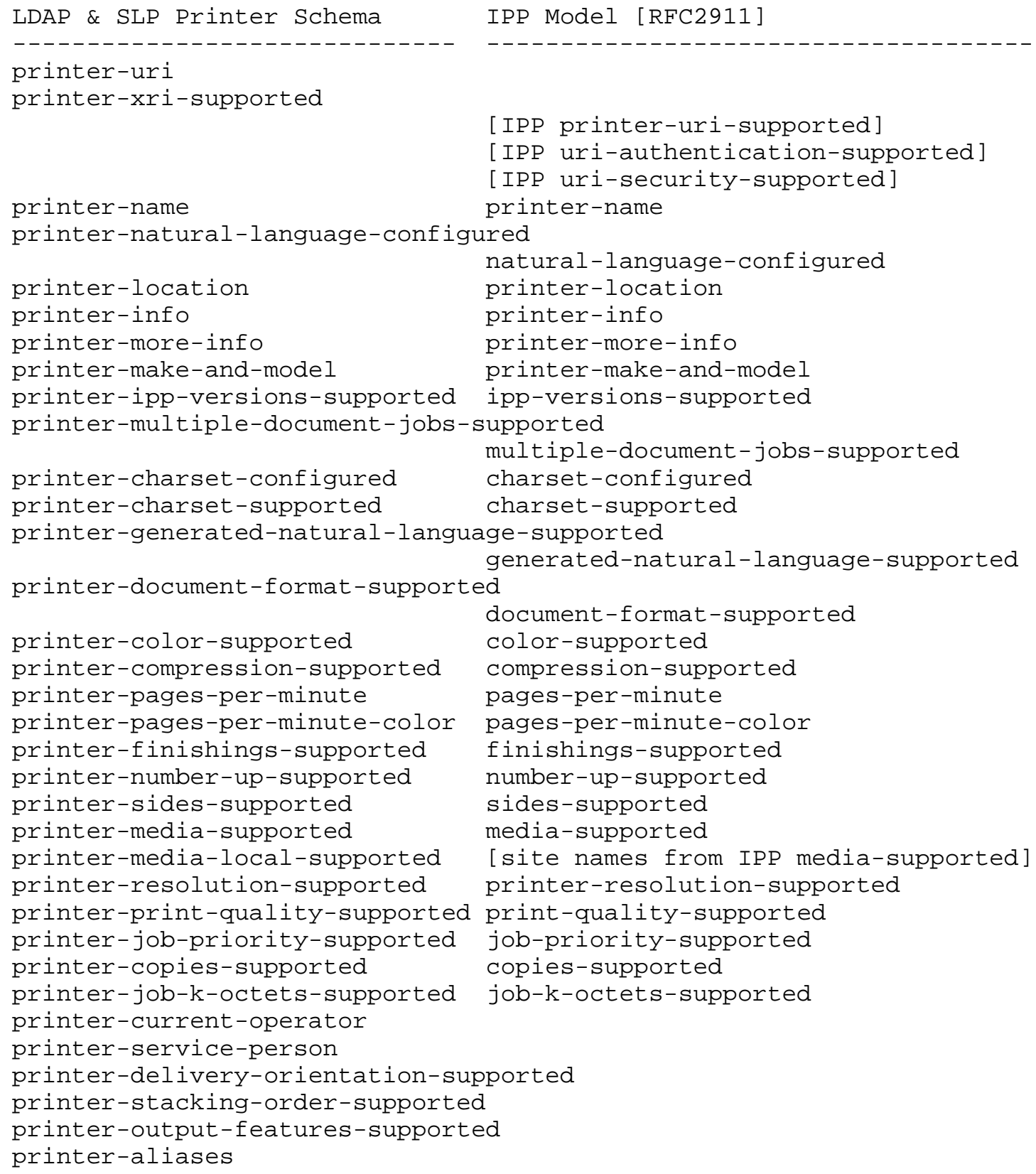


4.1. printer-uri

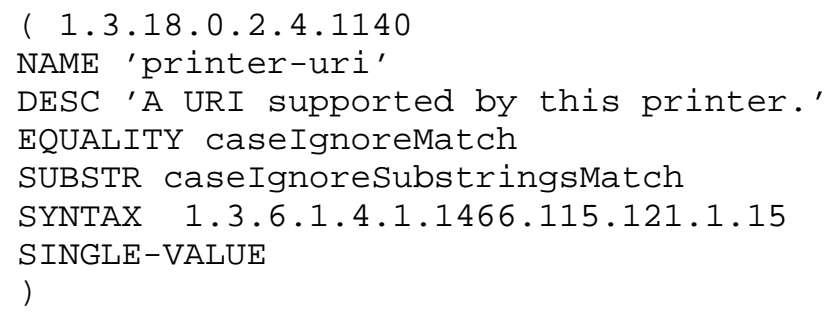


Note: LDAP application clients should not attempt to use malformed URI values read from this attribute. LDAP administrative clients should not write malformed URI values into this attribute.

Note: This attribute is based on 'printer-uri-supported', 'uriauthentication-supported', and ''uri-security-supported' (called the 'Three Musketeers' because they are parallel ordered attributes) defined in IPP/1.1 [RFC2911]. This attribute unfolds those IPP/1.1 attributes and thus avoids the ordering (and same number of values) constraints of the IPP/1.1 separate attributes.

Defined keywords for fields include:

'uri' (IPP 'printer-uri-supported')

'auth' (IPP 'uri-authentication-supported')

'sec' (IPP 'uri-security-supported')

A missing 'auth' field should be interpreted to mean 'none'. Per IPP/1.1 [RFC2911], defined values of the 'auth' field include:

'none' (no authentication for this URI)

'requesting-user-name' (from operation request)

'basic' (HTTP/1.1 Basic [RFC2617])

'digest' (HTTP/1.1 Basic, [RFC2617])

'certificate' (from certificate)

A missing 'sec' field should be interpreted to mean 'none'. Per IPP/1.1 [RFC2911], defined values of the 'sec' field include:

'none' (no security for this URI)

'ssl3' (Netscape SSL3)

'tls' (IETF TLS/1.0, [RFC2246])

Each XRI field should be delimited by ' $<$ '. For example:

' uri=ipp: //foo. com $<$ auth=digest $<$ sec $=$ t $l s<'$

' uri=lpr: //bar.com< auth=none< sec=none $<$ '

' uri=mailto:printerlfoobar. com $<$ auth=none $<$ sec=none $<$ '

Note: The syntax and delimiter for this attribute are aligned with the equivalent attribute in the 'service:printer:' v2.0 template [SLP-PRT]. Whitespace is permitted after (but not before) the delimiter ' $<$ '. Note that this delimiter differs from printerresolution-supported.

Note: See Sections 1.1, 1.2, and 1.3 for rationale for design choices. 
4.3. printer-name

$(1.3 .18 .0 .2 .4 .1135$

NAME 'printer-name'

DESC 'The site-specific administrative name of this printer.'

EQUALITY caseIgnorematch

SUBSTR caseIgnoreSubstringsMatch

SYNTAX 1.3.6.1.4.1.1466.115.121.1.15\{127\}

SINGLE-VALUE

)

Values of this attribute should be specified in the language specified in printer-natural-language-configured (for example, to support text-to-speech conversions), although the printer's name may be specified in any language. This name may be the last part of the printer's URI or it may be completely unrelated. This name may contain characters that are not allowed in a conventional URI (see [RFC2396]).

4.4. printer-natural-language-configured

$(1.3 .18 .0 .2 .4 .1119$

NAME 'printer-natural-language-configured'

DESC 'The configured natural language in which error and status messages will be generated (by default) by this printer.'

EQUALITY caseIgnoreMatch

SUBSTR caseIgnoreSubstringsMatch

SYNTAX 1.3.6.1.4.1.1466.115.121.1.15\{127\}

SINGLE-VALUE

)

Also, a possible natural language for printer string attributes set by operator, system administrator, or manufacturer. Also, the (declared) natural language of the printer-name, printer-location, printer-info, and printer-make-and-model attributes of this printer.

Values of language tags should conform to "Tags for the Identification of Languages" [RFC3066]. For example:

'en-us' (English as spoken in the US)

'fr-fr' (French as spoken in France)

For consistency with IPP/1.1 [RFC2911], language tags in this attribute should be lowercase normalized. 
4.5. printer-location

$(1.3 .18 \cdot 0.2 .4 .1136$

NAME 'printer-location'

DESC 'The physical location of this printer.'

EQUALITY caseIgnorematch

SUBSTR caseIgnoreSubstringsMatch

SYNTAX 1.3.6.1.4.1.1466.115.121.1.15\{127\}

SINGLE-VALUE

)

For example:

'Room 123A'

'Second floor of building XYZ'

4.6. printer-info

$(1.3 .18 \cdot 0.2 .4 .1139$

NAME 'printer-info'

DESC 'Descriptive information about this printer.'

EQUALITY caseIgnoreMatch

SUBSTR caseIgnoreSubstringsMatch

SYNTAX 1.3.6.1.4.1.1466.115.121.1.15\{127\}

SINGLE-VALUE

)

For example:

'This printer can be used for printing color transparencies for HR presentations'

'Out of courtesy for others, please print only small (1-5 page) jobs at this printer'

'This printer is going away on July 1, 1997, please find a new printer'

4.7. printer-more-info

$(1.3 .18 .0 .2 .4 .1134$

NAME 'printer-more-info'

DESC 'A URI for more information about this specific printer.'

EQUALITY caseIgnoreMatch

SUBSTR caseIgnoreSubstringsMatch

SYNTAX 1.3.6.1.4.1.1466.115.121.1.15

SINGLE-VALUE

) 
For example, this could be an HTTP type URI referencing an HTML page accessible to a Web Browser. The information obtained from this URI is intended for end user consumption.

Values of URI should conform to [RFC2396], although URI schemes may be defined which do not conform to [RFC2396] (see [RFC2717] and [RFC2718]).

Note: LDAP application clients should not attempt to use malformed URI values read from this attribute. LDAP administrative clients should not write malformed URI values into this attribute.

Note: See Sections 1.1, 1.2, and 1.3 for rationale for design choices.

4.8. printer-make-and-model

( 1.3 .18 .0 .2 .4 .1138

NAME 'printer-make-and-model'

DESC 'Make and model of this printer.'

EQUALITY caseIgnoreMatch

SUBSTR caseIgnoreSubstringsMatch

SYNTAX 1.3.6.1.4.1.1466.115.121.1.15\{127\}

SINGLE-VALUE

)

Note: The printer manufacturer may initially populate this attribute.

4.9. printer-ipp-versions-supported

$(1.3 .18 \cdot 0.2 \cdot 4.1133$

NAME 'printer-ipp-versions-supported'

DESC 'IPP protocol version(s) that this printer supports.'

EQUALITY caseIgnoreMatch

SUBSTR caseIgnoreSubstringsMatch

SYNTAX 1.3.6.1.4.1.1466.115.121.1.15\{127\}

)

The IPP protocol version(s) should include major and minor versions, i.e., the exact version numbers for which this Printer implementation meets the IPP version-specific conformance requirements. 
4.10. printer-multiple-document-jobs-supported

$(1.3 .18 \cdot 0.2 .4 .1132$

NAME 'printer-multiple-document-jobs-supported'

DESC 'Indicates whether or not this printer supports more than one document per job.'

EQUALITY booleanMatch

SYNTAX 1.3.6.1.4.1.1466.115.121.1.7

SINGLE-VALUE

)

4.11. printer-charset-configured

$(1.3 .18 .0 .2 .4 .1109$

NAME 'printer-charset-configured'

DESC 'The configured charset in which error and status messages will be generated (by default) by this printer.'

EQUALITY caseIgnorematch

SYNTAX 1.3.6.1.4.1.1466.115.121.1.15\{63\}

SINGLE-VALUE

)

Also, a possible charset for printer string attributes set by operator, system administrator, or manufacturer. For example:

'utf-8' (ISO 10646/Unicode in UTF-8 transform [RFC2279])

'iso-8859-1' (Latin1)

Values of charset tags should be defined in the IANA Registry of Coded Character Sets [IANA-CHAR] (see also [RFC2978]) and the ' (preferred MIME name)' should be used as the charset tag in this attribute.

For consistency with IPP/1.1 [RFC2911], charset tags in this attribute should be lowercase normalized.

4.12. printer-charset-supported

$(1.3 .18 .0 .2 .4 .1131$

NAME 'printer-charset-supported'

DESC ' Set of charsets supported for the attribute values of syntax Directorystring for this directory entry.' EQUALITY caseIgnoreMatch

SYNTAX 1.3.6.1.4.1.1466.115.121.1.15\{63\}

) 
For example:

'utf-8' (ISO 10646/Unicode in UTF-8 transform [RFC2279])

'iso-8859-1' (Latin1)

Values of charset tags should be defined in the IANA Registry of Coded Character Sets [IANA-CHAR] (see also [RFC2978]) and the

' (preferred MIME name)' should be used as the charset tag in this attribute.

For consistency with IPP/1.1 [RFC2911], charset tags in this attribute should be lowercase normalized.

4.13. printer-generated-natural-language-supported

$(1.3 .18 \cdot 0.2 .4 .1137$

NAME 'printer-generated-natural-language-supported'

DESC 'Natural language(s) supported for this directory entry.' EQUALITY caseIgnoreMatch

SUBSTR caseIgnoreSubstringsMatch SYNTAX 1.3.6.1.4.1.1466.115.121.1.15\{63\}

)

Values of language tags should conform to "Tags for the Identification of Languages" [RFC3066]. For example:

'en-us' (English as spoken in the US)

'fr-fr' (French as spoken in France)

For consistency with IPP/1.1 [RFC2911], language tags in this attribute should be lowercase normalized.

4.14. printer-document-format-supported

$(1.3 .18 .0 .2 .4 .1130$

NAME 'printer-document-format-supported'

DESC 'The possible source document formats which may be interpreted and printed by this printer.'

EQUALITY caseIgnoreMatch

SUBSTR caseIgnoreSubstringsMatch

SYNTAX 1.3.6.1.4.1.1466.115.121.1.15\{127\}

)

Values of document formats should be MIME media types defined in the IANA Registry of MIME Media Types [IANA-MIME] (see also [RFC2048]). 


\subsection{5. printer-color-supported}

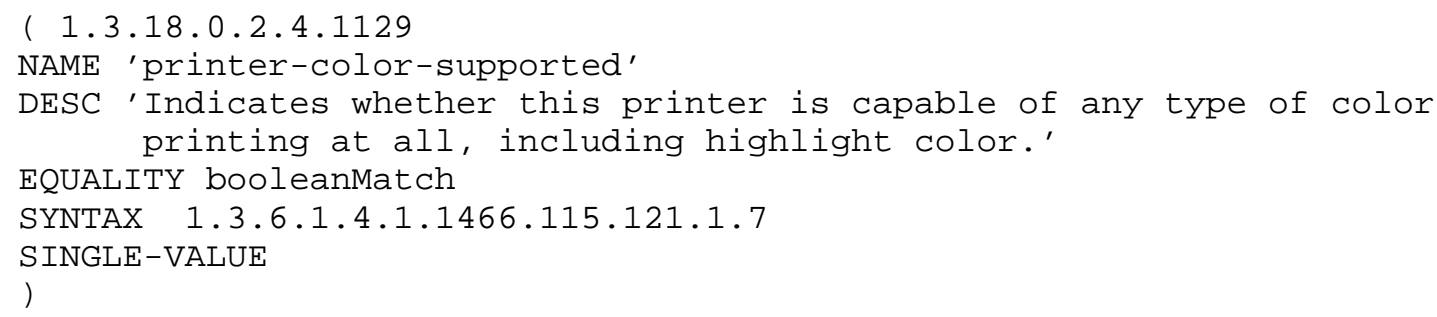

4.16. printer-compression-supported

( $1.3 .18 \cdot 0.2 .4 .1128$

NAME 'printer-compression-supported'

DESC 'Compression algorithms supported by this printer.' EQUALITY caseIgnoreMatch

4.17. printer-pages-per-minute

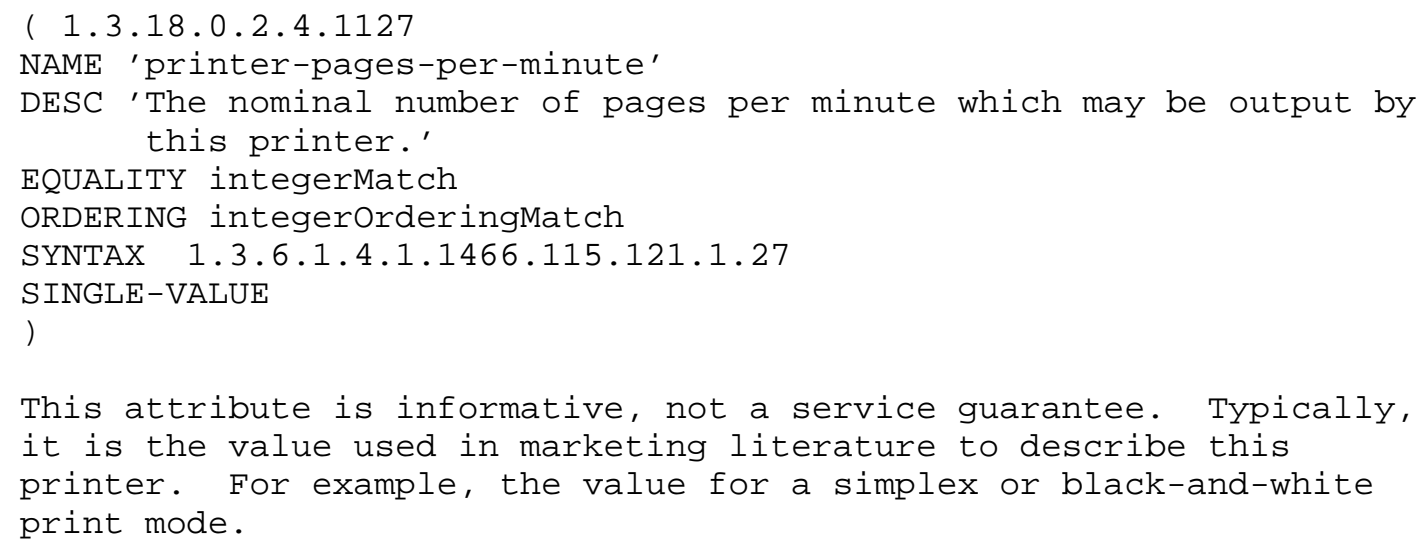


4.18. printer-pages-per-minute-color

$(1.3 .18 .0 .2 .4 .1126$

NAME 'printer-pages-per-minute-color'

DESC 'The nominal number of color pages per minute which may be output by this printer.'

EQUALITY integerMatch

ORDERING integerOrderingMatch

SYNTAX 1.3.6.1.4.1.1466.115.121.1.27

SINGLE-VALUE

)

This attribute is informative, not a service guarantee. Typically, it is the value used in marketing literature to describe this printer.

4.19. printer-finishings-supported

$(1.3 .18 \cdot 0.2 .4 .1125$

NAME 'printer-finishings-supported'

DESC 'The possible finishing operations supported by this printer.' EQUALITY caseIgnoreMatch

SUBSTR caseIgnoreSubstringsMatch

SYNTAX 1.3.6.1.4.1.1466.115.121.1.15\{255\}

)

Values defined in IPP/1.1 [RFC2911] include: 'none', 'staple',

'punch', 'cover', 'bind', 'saddle-stitch', 'edge-stitch',

'staple-top-left', 'staple-bottom-left', 'staple-top-right',

'staple-bottom-right', 'edge-stitch-left', ' edge-stitch-top',

'edge-stitch-right', 'edge-stitch-bottom', 'staple-dual-left',

'staple-dual-top', 'staple-dual-right', 'staple-dual-bottom' .

Note: Implementations may support other values.

4.20. printer-number-up-supported

$(1.3 .18 \cdot 0.2 \cdot 4.1124$

NAME 'printer-number-up-supported'

DESC 'The possible numbers of print-stream pages to impose upon a single side of an instance of a selected medium.'

EQUALITY integerMatch

ORDERING integerOrderingMatch

SYNTAX 1.3.6.1.4.1.1466.115.121.1.27

) 
Values defined in IPP/1.1 [RFC2911] include: '1', '2', and '4'.

Note: Implementations may support other values.

4.21. printer-sides-supported

( 1.3 .18 .0 .2 .4 .1123

NAME 'printer-sides-supported'

DESC 'The number of impression sides (one or two) and the two-sided impression rotations supported by this printer.'

EQUALITY caseIgnoreMatch

SYNTAX 1.3.6.1.4.1.1466.115.121.1.15\{127\}

)

Values defined in IPP/1.1 [RFC2911] include: 'one-sided', 'twosided-long-edge', 'two-sided-short-edge' .'

4.22. printer-media-supported

$(1.3 .18 \cdot 0 \cdot 2 \cdot 4 \cdot 1122$

NAME 'printer-media-supported'

DESC 'The standard names/types/sizes (and optional color suffixes) of the media supported by this printer.'

EQUALITY caseIgnoreMatch

SUBSTR caseIgnoreSubstringsMatch

SYNTAX 1.3.6.1.4.1.1466.115.121.1.15\{255\}

)

Values are defined in IPP/1.1 [RFC2911] or any IANA registered extensions. For example:

'iso-a4'

'envelope'

'na-letter-white'

4.23. printer-media-local-supported

$(1.3 .18 \cdot 0.2 \cdot 4.1117$

NAME 'printer-media-local-supported'

DESC 'Site-specific names of media supported by this printer.' EQUALITY caseIgnoreMatch

SUBSTR caseIgnoreSubstringsMatch

SYNTAX 1.3.6.1.4.1.1466.115.121.1.15\{255\}

)

Values should be in the natural language specified by printernatural-language-configured. 
For example:

'purchasing-form' (site-specific name)

as opposed to 'na-letter' (standard keyword from IPP/1.1 [RFC2911])

in the printer-media-supported attribute.

4.24. printer-resolution-supported

( $1.3 \cdot 18 \cdot 0 \cdot 2 \cdot 4 \cdot 1121$

NAME 'printer-resolution-supported'

DESC 'List of resolutions supported for printing documents by this printer.'

EQUALITY caseIgnoreMatch

SUBSTR caseIgnoreSubstringsMatch

SYNTAX 1.3.6.1.4.1.1466.115.121.1.15\{255\}

)

Each resolution value should be a string containing 3 fields:

1) Cross feed direction resolution (positive integer);

2) Feed direction resolution (positive integer);

3) Unit - 'dpi' (dots per inch) or 'dpcm' (dots per centimeter).

Each resolution field should be delimited by '>'. For example:

'300> 300> dpi>'

'600>600> dpi>'

Note: This attribute is based on 'printer-resolution-supported' defined in IPP/1.1 [RFC2911] (which has a binary complex encoding) derived from 'prtMarkerAddressabilityFeedDir', 'prtMarkerAddressabilityXFeedDir', and 'prtMarkerAddressabilityUnit' defined in the Printer MIB [RFC1759] (which have integer encodings).

Note: The syntax and delimiter for this attribute are aligned with the equivalent attribute in the 'service:printer:' v2.0 template [SLP-PRT]. Whitespace is permitted after (but not before) the delimiter ' $>$ '. Note that this delimiter differs from printer-xrisupported. 
4.25. printer-print-quality-supported

$(1.3 .18 \cdot 0.2 .4 .1120$

NAME 'printer-print-quality-supported'

DESC 'List of print qualities supported for printing documents on this printer.'

EQUALITY caseIgnorematch

SYNTAX 1.3.6.1.4.1.1466.115.121.1.15\{127\}

)

Values defined in IPP/1.1 [RFC2911] include:

'unknown'

'draft'

'normal'

'high'

4.26. printer-job-priority-supported

$(1.3 \cdot 18 \cdot 0 \cdot 2 \cdot 4 \cdot 1110$

NAME 'printer-job-priority-supported'

DESC 'Indicates the number of job priority levels supported by this printer.'

EQUALITY integerMatch

ORDERING integerOrderingMatch

SYNTAX 1.3.6.1.4.1.1466.115.121.1.27

SINGLE-VALUE

)

An IPP/1.1 [RFC2911] conformant Printer, which supports job priority, always supports a full range of priorities from '1' to '100' (to ensure consistent behavior), therefore this attribute describes the 'granularity' of priority supported. Values of this attribute are from '1' to' 100 '.

4.27. printer-copies-supported

$(1.3 .18 .0 .2 .4 .1118$

NAME 'printer-copies-supported'

DESC 'The maximum number of copies of a document that may be printed as a single job on this printer.'

EQUALITY integerMatch

ORDERING integerOrderingMatch

SYNTAX 1.3.6.1.4.1.1466.115.121.1.27

SINGLE-VALUE

) 
A positive value indicates the maximum supported copies. A value of ' 0 ' indicates no maximum limit. A value of '-1' indicates ' unknown'.

Note: The syntax and values for this attribute are aligned with the equivalent attribute in the 'service:printer:' v2.0 template [SLP$\mathrm{PRT}$ ] .

4.28. printer-job-k-octets-supported

$(1.3 \cdot 18 \cdot 0 \cdot 2 \cdot 4 \cdot 1111$

NAME 'printer-job-k-octets-supported'

DESC 'The maximum size in kilobytes (1,024 octets actually) incoming print job that this printer will accept.'

EQUALITY integerMatch

ORDERING integerOrderingMatch

SYNTAX 1.3.6.1.4.1.1466.115.121.1.27

SINGLE-VALUE

)

A positive value indicates the maximum supported job size. A value of ' 0 ' indicates no maximum limit. A value of '-1' indicates

' unknown'.

Note: The syntax and values for this attribute are aligned with the equivalent attribute in the 'service:printer:' v2.0 template [SLPPRT ].

4.29. printer-current-operator

( $1.3 .18 \cdot 0 \cdot 2 \cdot 4 \cdot 1112$

NAME 'printer-current-operator'

DESC 'The identity of the current human operator responsible for operating this printer.'

EQUALITY caseIgnoreMatch

SUBSTR caseIgnoreSubstringsMatch

SYNTAX 1.3.6.1.4.1.1466.115.121.1.15\{127\}

SINGLE-VALUE

)

The value of this attribute should include information that would enable other humans to reach the operator, such as a telephone number. 
4.30. printer-service-person

$(1.3 .18 \cdot 0.2 .4 .1113$

NAME 'printer-service-person'

DESC 'The identity of the current human service person responsible for servicing this printer.'

EQUALITY caseIgnoreMatch

SUBSTR caseIgnoreSubstringsMatch

SYNTAX 1.3.6.1.4.1.1466.115.121.1.15\{127\}

SINGLE-VALUE

)

The value of this attribute should include information that would enable other humans to reach the service person, such as a telephone number.

4.31. printer-delivery-orientation-supported

$(1.3 .18 \cdot 0.2 \cdot 4.1114$

NAME 'printer-delivery-orientation-supported'

DESC 'The possible delivery orientations of pages as they are printed and ejected from this printer.'

EQUALITY caseIgnoreMatch

SYNTAX 1.3.6.1.4.1.1466.115.121.1.15\{127\}

)

Values defined include:

' unknown'

'face-up'

'face-down'

Note: The syntax and values for this attribute are aligned with the equivalent attribute in the 'service:printer:' v2.0 template [SLPPRT ] .

4.32. printer-stacking-order-supported

$(1.3 .18 .0 .2 .4 .1115$

NAME 'printer-stacking-order-supported'

DESC 'The possible stacking order of pages as they are printed and ejected from this printer.'

EQUALITY caseIgnoreMatch

SYNTAX 1.3.6.1.4.1.1466.115.121.1.15\{127\}

) 
Values defined include:

' unknown'

'first-to-last'

'last-to-first'

Note: The syntax and values for this attribute are aligned with the equivalent attribute in the 'service:printer:' v2.0 template [SLP$\mathrm{PRT}$ ] .

4.33. printer-output-features-supported

$(1.3 .18 \cdot 0.2 .4 .1116$

NAME 'printer-output-features-supported'

DESC 'The possible output features supported by this printer.' EQUALITY caseIgnoreMatch

SYNTAX 1.3.6.1.4.1.1466.115.121.1.15\{127\}

)

Values defined include:

' unknown'

'bursting'

'decollating'

'page-collating'

'offset-stacking'

Note: The syntax and values for this attribute are aligned with the equivalent attribute in the 'service:printer:' v2.0 template [SLP$\mathrm{PRT}$ ].

Note: Implementations may support other values.

4.34. printer-aliases

( 1.3 .18 .0 .2 .4 .1108

NAME 'printer-aliases'

DESC 'List of site-specific administrative names of this printer in addition to the value specified for printer-name.'

EQUALITY caseIgnoreMatch

SUBSTR caseIgnoreSubstringsMatch

SYNTAX 1.3.6.1.4.1.1466.115.121.1.15\{127\}

)

Values of this attribute should be specified in the language specified in printer-natural-language-configured (for example, to support text-to-speech conversions), although the printer's alias may be specified in any language. 
5. Definition of Syntaxes

No new attribute syntaxes are defined by this document.

The attribute types defined in section 4 of this document reference syntax OIDs defined in section 6 of [RFC2252], which are summarized below:

$\begin{array}{lll}\text { Syntax OID } & \text { Syntax Description } \\ --1.6 .1 .4 .1 .1466 .115 .121 .1 .7 & \text { Boolean } \\ 1.3 .6 & & \\ 1.3 .6 .1 .4 .1 .1466 .115 .121 .1 .15 & \text { DirectoryString (UTF-8 [RFC2279]) } \\ 1.3 .6 .1 .4 .1 .1466 .115 .121 .1 .27 & \text { Integer }\end{array}$

6. Definition of Matching Rules

No new matching rules are defined by this document.

The attribute types defined in Section 4 of this document reference matching rules defined in section 8 of [RFC2252] and/or Section 2 of [RFC3698], which are summarized below:

$\begin{array}{lll}\text { Matching Rule OID } & \text { Matching Rule Name } & \text { Usage } \\ ----------- & ---- \\ 2.5 .13 .13 & -------------- & \text { EQUALITY } \\ 2.5 .13 .2 & \text { booleanMatch } & \text { EQUALITY } \\ 2.5 .13 .14 & \text { caseIgnoreMatch } & \text { EQUALITY } \\ 2.5 .13 .15 & \text { integerMatch } & \text { ORDERING } \\ 2.5 .13 .4 & \text { integerOrderingMatch } & \text { SUBSTR }\end{array}$

7. IANA Considerations

This document does not define any new syntaxes or matching rules.

This document does define the following object Identifier

Descriptors. They have been registered by the IANA:

7.1. Registration of Object Classes

Subject: Request for LDAP Descriptor Registration

Descriptor (short name): see table below

Object Identifier: see table below

Person \& email address to contact for further information: see below

Usage: object class 


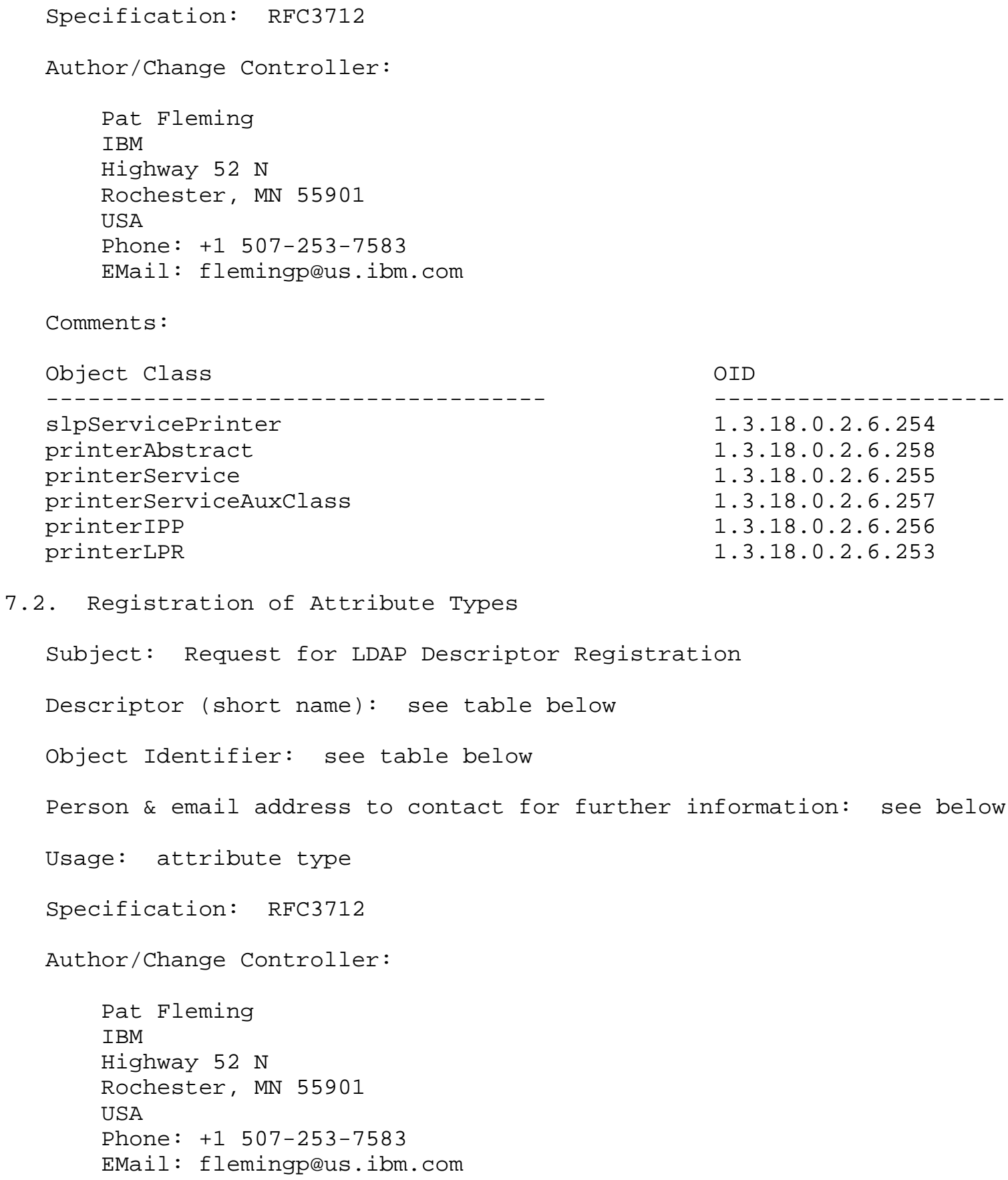

7.2. Registration of Attribute Types

Subject: Request for LDAP Descriptor Registration

Descriptor (short name): see table below

Object Identifier: see table below

Person \& email address to contact for further information: see below

Usage: attribute type

Specification: RFC3712

Author/Change Controller:

Pat Fleming

IBM

Highway $52 \mathrm{~N}$

Rochester, MN 55901

USA

Phone: +1 507-253-7583

EMail: flemingpeus.ibm.com 
Comments :

Attribute Type

printer-uri

printer-xri-supported

printer-name

printer-natural-language-configured

printer-location

printer-info

printer-more-info

printer-make-and-model

printer-ipp-versions-supported

printer-multiple-document-jobs-supported

printer-charset-configured

printer-charset-supported

printer-generated-natural-language-supported

printer-document-format-supported

printer-color-supported

printer-compression-supported

printer-pages-per-minute

printer-pages-per-minute-color

printer-finishings-supported

printer-number-up-supported

printer-sides-supported

printer-media-supported

printer-media-local-supported

printer-resolution-supported

printer-print-quality-supported

printer-job-priority-supported

printer-copies-supported

printer-job-k-octets-supported

printer-current-operator

printer-service-person

printer-delivery-orientation-supported

printer-stacking-order-supported

printer-output-features-supported

printer-aliases
OID

$1 \cdot 3 \cdot 18 \cdot 0 \cdot 2 \cdot 4 \cdot 1140$

$1.3 .18 \cdot 0.2 .4 .1107$

$1 \cdot 3 \cdot 18 \cdot 0 \cdot 2 \cdot 4 \cdot 1135$

$1.3 \cdot 18 \cdot 0 \cdot 2 \cdot 4 \cdot 1119$

1.3 .18 .0 .2 .4 .1136

$1.3 .18 \cdot 0.2 \cdot 4.1139$

$1.3 .18 \cdot 0.2 \cdot 4.1134$

$1.3 .18 \cdot 0.2 .4 .1138$

$1.3 .18 \cdot 0.2 .4 .1133$

1.3 .18 .0 .2 .4 .1132

1.3 .18 .0 .2 .4 .1109

1.3 .18 .0 .2 .4 .1131

$1.3 .18 \cdot 0.2 .4 .1137$

$1.3 .18 \cdot 0.2 .4 .1130$

1.3 .18 .0 .2 .4 .1129

$1.3 .18 \cdot 0.2 .4 .1128$

1.3 .18 .0 .2 .4 .1127

1.3 .18 .0 .2 .4 .1126

1.3 .18 .0 .2 .4 .1125

$1.3 .18 \cdot 0.2 .4 .1124$

$1.3 .18 \cdot 0.2 .4 .1123$

$1.3 .18 \cdot 0.2 \cdot 4.1122$

1.3 .18 .0 .2 .4 .1117

$1.3 .18 \cdot 0.2 .4 .1121$

$1.3 .18 \cdot 0.2 .4 .1120$

$1.3 .18 \cdot 0.2 .4 .1110$

$1.3 .18 \cdot 0.2 .4 .1118$

1.3 .18 .0 .2 .4 .1111

1.3 .18 .0 .2 .4 .1112

$1.3 .18 \cdot 0.2 \cdot 4.1113$

1.3 .18 .0 .2 .4 .1114

1.3 .18 .0 .2 .4 .1115

$1.3 .18 \cdot 0.2 .4 .1116$

1.3 .18 .0 .2 .4 .1108

8. Internationalization Considerations

All text string attributes defined in this document of syntax [RFC2279], as required by [RFC2252].

A language tag [RFC3066] for all of the text string attributes defined in this document is contained in the printer-naturallanguage-configured attribute. 
Therefore, all object classes defined in this document conform to the "IETF Policy on Character sets and Languages" [RFC2277].

9. Security Considerations

See [RFC2829] for detailed guidance on authentication methods for LDAP. See [RFC2830] for detailed guidance of using TLS/1.0 [RFC2246] to supply connection confidentiality and data integrity for LDAP sessions.

As with any LDAP schema, it is important to protect specific entries and attributes with the appropriate access control. It is particularly important that only administrators can modify entries defined in this LDAP printer schema. Otherwise, an LDAP client might be fooled into diverting print service requests from the original printer (or spooler) to a malicious intruder's host system, thus exposing the information in printed documents.

For additional security considerations of deploying printers in an IPP environment, see Section 8 of [RFC2911].

10. References

10.1. Normative References

[IANA-CHAR] IANA Registry of Character sets http://www.iana.org/assignments/charset-reg/...

[IANA-MIME] IANA Registry of MIME Media Types http://wWw.iana.org/assignments/media-types/...

[LDAP-TS] Hodges, J. and R. Morgan, "Lightweight Directory Access Protocol (v3): Technical Specification", RFC 3377, September 2002 .

[RFC1274] Barker, P. and S. Kille, "The COSINE and Internet X.500 Schema", RFC 1274, November 1991.

[RFC1759] Smith, R., Wright, F., Hastings, T., Zilles, S. and J. Gyllenskog, "Printer MIB", RFC 1759, March 1995.

[RFC2119] Bradner, S., "Key words for use in RFCs to Indicate Requirement Levels", BCP 14, RFC 2119, March 1997.

[RFC2252] Wahl, M., Coulbeck, T., Howes, T. and S. Kille, "Lightweight Directory Access Protocol (v3): Attribute Syntax Definitions", RFC 2252, December 1997. 
[RFC2396] Berners-Lee. T., Fielding, R. and L. Masinter, "URI Generic Syntax", RFC 2396, August 1998.

[RFC2829] Wahl, M., Alvestrand, H., Hodges, J. and R. Morgan, "Authentication Methods for LDAP", RFC 2829, May 2000.

[RFC2830] Hodges, J., Morgan, R. and M. Wahl, "Lightweight Directory Access Protocol (v3): Extension for Transport Layer security", RFC 2830, May 2000.

[RFC2911] Hastings, T., Ed.., Herrito, R., deBry, R., Isaacson, S. and P. Powell, "Internet Printing Protocol/1.1: Model and Semantics", RFC 2911, September 2000.

[RFC2926] Kempf, J., Moats, R. and P. St. Pierre, "Conversion of LDAP Schemas to and from SLP Templates", RFC 2926, September 2000 .

[RFC3066] Alvestrand, H., "Tags for the Identification of Languages", BCP 47, RFC 3066, January 2001.

[RFC3698] Zeilenga, K., Ed., "Lightweight Directory Access Protocol (LDAP): Additional Matching Rules", RFC 3698, February 2004 .

10.2. Informative References

[IANA-SLPT] IANA Registry of SLP Templates http://www.iana.org/assignments/svrloc-templates/...

[RFC1179] McLaughlin, L., "Line Printer Daemon Protocol", RFC 1179, August 1990 .

[RFC1951] Deutsch, P., "DEFLATE Compressed Data Format Specification Version 1.3", RFC 1951, May 1996.

[RFC1952] Deutsch, P., "GZIP File Format Specification Version 4.3", RFC 1952, May 1996.

[RFC1977] Schryver, V., "PPP BSD Compression Protocol", RFC 1977, August 1996 .

[RFC2048] Freed, N., Klensin, J. and J. Postel, "Multipurpose Internet Mail Extensions (MIME) Part Four: Registration Procedures", BCP 13, RFC 2048, November 1996. 
[RFC2079] Smith, M., "Definition of an X.500 Attribute Type and an object Class to Hold Uniform Resource Identifiers (URIs)", RFC 2079, January 1997.

[RFC2246] Dierks, T. and C. Allen, "TLS Protocol Version 1.0", RFC 2246, January 1999.

[RFC2277] Alvestrand, H., "IETF Policy on Character sets and Languages", RFC 2277, January 1998.

[RFC2279] Yergeau, F., "UTF-8, a Transformation Format of ISO 10646", RFC 2279, January 1998.

[RFC2608] Guttman, E., Perkins, C., Veizades, J. and M. Day, "Service Location Protocol v2", RFC 2608, June 1999.

[RFC2609] Guttman, E., Perkins, C. and J. Kempf, "Service Templates and Service: Schemes", RFC 2609, June 1999.

[RFC2617] Franks, J., Hallam-Baker, P., Hostetler, J., Lawrence, S., Leach, P., Luotonen, A. and L. Stewart, "HTTP Authentication: Basic and Digest Access Authentication", RFC 2617, June 1999.

[RFC2717] Petke, R. and I. King, "Registration Procedures for URL Scheme Names", RFC 2717, November 1999.

[RFC2718] Masinter, L., Alvestrand, H., Zigmond, D. and R. Petke, "Guidelines for new URL Schemes", BCP 19, RFC 2718, November 1999.

[RFC2978] Freed, N. and J.Postel, "IANA Charset Registration Procedures", RFC2978, October 2000 .

[SLP-PRT] St. Pierre, Isaacson, McDonald. Definition of the Printer Abstract Service Type v2.0, <durable URL below>, May 2000. Reviewed and approved by IETF SLP Designated Expert, according to Section 5 'IANA Considerations' in [RFC2609].

Archived in the IANA Registry of SLP Templates [IANASLPT] at: http://www.iana.org/assignments/svrloctemplates/printer.2.0.en

[W3C-IRI] Duerst, Suignard, "Internationalized Resource Identifiers (IRI), Work in Progress. 


\section{Acknowledgments}

The editors wish to acknowledge the very significant contributions of Ken Jones (Bytemobile) and Harry Lewis (IBM) during the development of this document.

Thanks to Patrik Faltstrom (Cisco), Ryan Moats (Lemur Networks), Robert Moore (IBM), Lee Rafalow (IBM), Kimberly Reger (IBM), Kurt Zeilenga (OpenLDAP), and the members of the IETF IPP Working Group, for review comments and help in preparing this document.

12. Authors' Addresses

Please send comments to the authors at the addresses listed below.

Pat Fleming

IBM

Highway $52 \mathrm{~N}$

Rochester, MN 55901

USA

Phone: +1 507-253-7583

EMail: flemingpaus.ibm.com

Ira McDonald

High North Inc

221 Ridge Ave

Grand Marais, MI 49839

USA

Phone: +1 906-494-2434

Email: imcdonaldesharplabs.com 
13. Full Copyright statement

Copyright (C) The Internet Society (2004). This document is subject to the rights, licenses and restrictions contained in $\mathrm{BCP} 78$ and except as set forth therein, the authors retain all their rights.

This document and the information contained herein are provided on an "AS IS" basis and THE CONTRIBUTOR, THE ORGANIZATION HE/SHE REPRESENTS OR IS SPONSORED BY (IF ANY), THE INTERNET SOCIETY AND THE INTERNET ENGINEERING TASK FORCE DISCLAIM ALL WARRANTIES, EXPRESS OR IMPLIED, INCLUDING BUT NOT LIMITED TO ANY WARRANTY THAT THE USE OF THE INFORMATION HEREIN WILL NOT INFRINGE ANY RIGHTS OR ANY IMPLIED WARRANTIES OF MERCHANTABILITY OR FITNESS FOR A PARTICULAR PURPOSE.

Intellectual Property

The IETF takes no position regarding the validity or scope of any Intellectual property Rights or other rights that might be claimed to pertain to the implementation or use of the technology described in this document or the extent to which any license under such rights might or might not be available; nor does it represent that it has made any independent effort to identify any such rights. Information on the procedures with respect to rights in RFC documents can be found in BCP 78 and BCP 79 .

Copies of IPR disclosures made to the IETF Secretariat and any assurances of licenses to be made available, or the result of an attempt made to obtain a general license or permission for the use of such proprietary rights by implementers or users of this specification can be obtained from the IETF on-line IPR repository at http://www.ietf.org/ipr.

The IETF invites any interested party to bring to its attention any copyrights, patents or patent applications, or other proprietary rights that may cover technology that may be required to implement this standard. Please address the information to the IETF at ietf-ipreietf.org.

Acknowledgement

Funding for the RFC Editor function is currently provided by the Internet Society. 